\title{
Publisher Correction: Hertz-linewidth semiconductor lasers using CMOS-ready ultra-high- $Q$ microresonators
}

Warren Jin (D), Qi-Fan Yang, Lin Chang, Boqiang Shen (D), Heming Wang (D), Mark A. Leal (D), Lue Wu (D), Maodong Gao, Avi Feshali, Mario Paniccia, Kerry J. Vahala (iD and John E. Bowers (D)

Correction to: Nature Photonics https://doi.org/10.1038/s41566-021-00761-7, published online 11 February 2021.

In the version of this Article originally published, the configuration of the external-cavity 4,000-Hz linewidth laser was given incorrectly in Table 1 as 'Hybrid III-V/Si $\mathrm{N}_{4}$ '; it should have been 'Heterogeneous III-V/Si $\mathrm{N}_{3}$ '. This has now been corrected in all versions of the Article.

Published online: 6 April 2021

https://doi.org/10.1038/s41566-021-00805-y

(C) The Author(s), under exclusive licence to Springer Nature Limited 2021 